\title{
Interdisciplinarity and insularity in the diffusion of knowledge: an analysis of disciplinary boundaries between philosophy of science and the sciences
}

\author{
John McLevey ${ }^{1}$ (D) Alexander V. Graham ${ }^{2} \cdot$ Reid Mcllroy-Young ${ }^{3} \cdot$ \\ Pierson Browne $^{2} \cdot$ Kathryn S. Plaisance ${ }^{4}$
}

Received: 11 February 2018

(C) Akadémiai Kiadó, Budapest, Hungary 2018

\begin{abstract}
Two fundamentally different perspectives on knowledge diffusion dominate debates about academic disciplines. On the one hand, critics of disciplinary research and education have argued that disciplines are isolated silos, within which specialists pursue inward-looking and increasingly narrow research agendas. On the other hand, critics of the silo argument have demonstrated that researchers constantly import and export ideas across disciplinary boundaries. These perspectives have different implications for how knowledge diffuses, how intellectuals gain and lose status within their disciplines, and how intellectual reputations evolve within and across disciplines. We argue that highly general claims about the nature of disciplinary boundaries are counterproductive, and that research on the nature of specific disciplinary boundaries is more useful. To that end, this paper uses a novel publication and citation network dataset and statistical models of citation networks to test hypotheses about the boundaries between philosophy of science and 11 disciplinary clusters. Specifically, we test hypotheses about whether engaging with and being cited by scientific communities outside philosophy of science has an impact on one's position within philosophy of science. Our results suggest that philosophers of science produce interdisciplinary scholarship, but they tend not to cite work by other philosophers when it is published in journals outside of their discipline. Furthermore, net of other factors, receiving citations from other disciplines has no meaningful impact-positive or negative-on citations within philosophy of science. We conclude by considering this evidence for simultaneous interdisciplinarity and insularity in terms of scientific trading theory and other work on disciplinary boundaries and communication.
\end{abstract}

Keywords Disciplines - Intellectual networks - Exponential random graph models · Diffusion - Citations - Sociology of science $\cdot$ Science of science $\cdot$ Philosophy of science

John McLevey

john.mclevey@uwaterloo.ca

Extended author information available on the last page of the article 


\section{Introduction}

Two fundamentally different perspectives on knowledge diffusion dominate debates about academic disciplines. On the one hand, critics of disciplinary research and education have argued that disciplines are isolated silos, within which specialists pursue inward-looking and increasingly narrow research agendas (e.g., Frodeman 2010). These silos are reproduced in part by the incommensurability of disciplinary paradigms and epistemic cultures, "disciplinary ethnocentrism" (Campbell 1969), asymmetrical borrowing (Klein 1990), and "willful ignorance," as researchers simultaneously make their own disciplinary knowledge more esoteric and push back against knowledge from other disciplines (see Jacobs 2014). Many interdisciplinary fields in the social sciences and humanities emerged in the context of these and related critiques of the disciplinary system (Ilhan 2013; Rojas 2007; Small 1999; Turk-Bicakci 2007).

On the other hand, critics of the silo argument have demonstrated that researchers constantly import and export ideas across disciplinary boundaries (Jacobs 2014; Jacobs and Frickel 2009). An enormous volume of bibliometric evidence supports this position, from maps of science highlighting connections within and across disciplines (Boyack, Klavans, and Börner 2005; Börner, Chen, and Boyack 2003; Börner et al. 2012; Chen and Hicks 2004; Leydesdorff and Rafols 2009; Moody and Light 2006; Skupin, Biberstine, and Börner 2013) to quantitative research on diffusion, collaboration, and the exponential growth of science (Coccia and Bozeman 2016; Coccia and Wang 2016; Crane 1972; Liu et al. 2015; Price 1963; Yu et al. 2010; Zhu and Yan 2015). Theories, measures, and models of disciplinary knowledge diffusion (Bettencourt et al. 2008; Gao and Guan 2011; Herrera, Roberts, and Gulbahce 2010; Vitanov and Ausloos 2012; Yan 2014; Yan et al. 2013) and interdisciplinarity (Chen et al. 2014, 2015; Cronin and Sugimoto 2014; Larivière et al. 2014; Light and Adams 2016) are increasingly common. At the same time, qualitative research has advanced our understanding of how communication and collaboration across disciplinary boundaries is facilitated by trading zones and evolving professional languages (e.g., Collins 2011; Collins et al. 2010; Galison 1997; Gorman 2002, 2010).

A considerable amount of research on discipline-level knowledge diffusion has been guided by an economic metaphor: trade (Cronin and Meho 2008; Cronin and Pearson 1990; Larivière et al. 2012; Lockett and McWilliams 2005; Stigler 1994; Yan et al. 2013). Yan et al. (2013) systematically develop the metaphor, arguing that research on disciplinary knowledge imports and exports enables researchers to assess how "self-dependent" a discipline is, and what its "scientific trading impact" is within the global scientific system. This work focuses primarily on the macro-level trade states of disciplinary and interdisciplinary fields themselves, not on the concrete flows of knowledge across disciplines or on the actual boundaries between fields. This has led Yan (2014) to develop path-based approaches for identifying influential knowledge flows. Specifically, he uses maximum spanning tree algorithms to identify critical knowledge channels in weighted citation networks. This work moves beyond examining disciplinary import/export ratios by taking the network structure—or "backbone" (Boyack et al. 2005)—of science into account, and by emphasizing the positions that disciplines occupy along critical knowledge diffusion paths. In short, it reveals the relationships between disciplines by identifying the most important and well-traveled knowledge paths. 
All of this research is predominantly focused on the natural, life, and social sciences. The humanities are rarely included. ${ }^{1}$ As a result, we know much less about disciplinary boundaries and knowledge diffusion within the humanities, or between humanities and science disciplines. When it comes to disciplinary divides, we would naturally expect to see the most extreme disconnection between humanities and sciences disciplines. In his classic lecture from 1959, CP Snow suggested that the literary intellectuals representing the humanities not only had a different culture and language than those representing the physical sciences, but also that the two were deeply suspicious of one another and regarded one another as naive or misguided - albeit for different reasons (Snow 1959). Therefore, if disciplines are balkanized as a result of incommensurable paradigms or "willful ignorance," we would expect those who cross disciplinary boundaries between the humanities and the sciences to be the most affected.

In this paper, we focus on the boundaries between philosophy of science and the sciences that philosophers study. Our work starts from the assumption that either/or arguments about knowledge diffusion and disciplinarity (i.e., silo vs. web arguments) are counterproductive. Disciplinary researchers do import and export ideas, but they do not trade with all disciplines equally. The important question, then, is not whether disciplines are silos or connected in evolving social and intellectual webs in general. Rather, the important questions are in the formation, strength, and dissolution of the connections between specific disciplines and interdisciplines (see Van Leeuwen and Robert 2000), and in the relationship between intellectual reputations, status, and the shifting landscape of disciplinary boundaries. To that end, our specific goal in this paper is to better understand how engagement with and recognition from scientific fields impacts one's citations within philosophy of science. We use a statistical network model designed to estimate the probability of a philosophy of science paper receiving a citation from other philosophers of science, with citation counts in a wide-range of scientific disciplines as covariates. ${ }^{2}$ This enables us to test hypotheses (discussed below) about the nature of the boundaries between philosophy of science and the sciences it studies, on citation dynamics within philosophy of science.

There has been very little quantitative research on philosophy of science to date. This is especially true for bibliometric research. The only articles about philosophy of science in the core bibliometric, scientometric, and informetric journals are attempts to identify high impact historical publications (Wray and Bornmann 2015), or the relationships with cognate fields like epistemology (Kreuzman 2001). No studies have yet attempted to analyze the relationship between philosophy of science and the sciences, despite the potential to advance research on knowledge diffusion and disciplinary boundaries.

Qualitative research on philosophy emphasizes the cultural differences between philosophy and other disciplines. For example, Lamont's (2009) extensive study of decision making on grant adjudication committees in the social sciences and humanities identifies a variety of ways that philosophers and non-philosophers understand the discipline as being fundamentally different, and a "problem case" (pages 64-69). Other social scientific research on philosophy has mostly focused on the social structure of creativity and

\footnotetext{
${ }^{1}$ One legitimate reason for excluding the humanities is that available bibliometric data has less coverage. Books are more important in the humanities than in the social sciences, and books are not well indexed.

2 More specifically, we use Exponential Random Graph Models (ERGMs), which are relatively new development in network analysis (Lusher et al. 2012; Robins et al. 2007). ERGMs are rare in the sociology of science and information science, but researchers are starting to use them in bibliometric (Fanelli and Glänzel 2013) and knowledge management research (Jiang et al. 2015; Lungeanu et al. 2014; Su and Contractor 2011; Škerlavaj et al. 2010).
} 
interacting schools of thought (e.g., Collins' 2009), or on intellectual self-concepts (Gross 2002, 2009).

We recently conducted an international survey of philosophers of science (which will be reported in other articles) that suggests that the majority of philosophers of science want their work to have an influence on science, but that being embedded in traditional philosophy departments makes it difficult to meaningfully engage with scientists. For example, one obvious way philosophers can influence science is to publish in scientific journals, yet our survey and interview data suggest that many philosophy departments tend not to recognize or reward publications outside philosophy journals. We examined this problem in greater depth in semi-structured interviews with philosophers of science from Canada, the United States, the United Kingdom, France, Germany, and Australia.

This perceived tendency not to recognize work that falls outside of the discipline poses unique challenges for philosophers of science in general, but especially for those who think that philosophy is better and more useful when it is engaged with, even embedded in, scientific communities (e.g., Fehr and Plaisance 2010). Proponents of this position are concerned that their engagement with science could ultimately hurt their academic careers, or those of their students (Fehr and Plaisance 2010). As such, advice from senior scholars varies from "don't do it" (or at least, wait until tenure) to "have dual careers," one philosophical, one scientific.

In the sections below, we describe four competing hypotheses about the boundaries between philosophy of science and the sciences. Then, we explain how we collected the raw data, constructed the network dataset, and developed the statistical models. We then present the findings and conclude with a discussion of the implications for research on disciplines, and for philosophers of science who wish to have an impact on the sciences they study.

\section{Hypotheses}

If the paradigms and epistemic cultures between philosophy of science and the sciences are radically mismatched, or if philosophers are insular and actively resist importing and exporting ideas-as hard critics of the disciplinary "silos" or critics of the siloed nature of philosophy itself suggest-, then we should expect increases in citations from the sciences to have a negative impact on citations within philosophy itself. The basic idea is that the more a philosopher is engaged with and appealing to scientists, the less appealing or relevant she is to philosophers. Her colleagues are less likely to see her as a "real" philosopher. Even further, it may be the case that, because of mismatched cultures, philosophers of science are actually penalized for engaging the sciences, especially controversial sciences such as behavioral genetics (see Panofsky 2014), where philosophers often demand "critical distance." In short, we would expect philosophers with higher citations in the sciences to have lower citations in philosophy of science.

Alternatively, if philosophers collectively value sharing their expertise with scientists, then we would not expect to see them penalized for successfully engaging scientific communities. Instead, we would expect citations from scientific fields to contribute to an increase the probability of receiving citations from philosophers as well. This could be due to cumulative advantage, for example, where citations beget more citations in a fashion familiar to any sociologist or information scientist since Merton's (1973) pioneering research. In short, success in one field begets success in others. If it is the case, we would expect to see philosophers that publish in scientific journals and get cited by scientists to 
have a higher probability of being cited by other philosophers. Among other things, this outcome could be due to a tendency for philosophically-minded scientists to cite philosophers that are well-respected within the philosophy of science community, or to a tendency for respected philosophers of science to invest in their intellectual reputations and visibility in the sciences by publishing in science journals.

Another possibility is that philosophers of science may differentiate between scientific disciplines, with some being considered worthy of serious engagement and others more deserving of "critical distance." Perhaps citations from disciplines that are broadly friendly to philosophical work - say some branches of cognitive science-may actually increase the likelihood of citations from within philosophy, whereas citations from more controversial fields - again, such as behavioral genetics-may decrease the likelihood of citations. In short, the likelihood of receiving a citation boost or a citation penalty varies by scientific field.

Finally, it is possible that most philosophers of science simply do not read extensively outside of their own discipline, and that writing in or being cited in scientific journals does not affect one's position with philosophy of science. If this is the case, we would expect to find that publishing in scientific journals has no significant positive or negative impact on a philosopher's chances of being cited by other philosophers.

To summarize, we assess the boundaries between philosophy of science and the sciences by testing four hypotheses:

1. Net of other factors, publishing in and being cited in science journals reduces the likelihood of being cited by other philosophers of science. We will refer to this as the citation penalty hypothesis.

2. Net of other factors, publishing in and being cited in science journals increases the likelihood of being cited by other philosophers. We will refer to this as the cumulative advantage hypothesis.

3. Net of other factors, the positive or negative effects of publishing or being cited in any in science journals varies based on the type of science from which a philosopher receives uptake. We will refer to this as the field-specific hypothesis.

4. Net of other factors, publishing in and being cited in science journals does not change the likelihood of being cited by other philosophers. We will refer to this as the separate worlds hypothesis.

\section{Data and methods}

Testing these hypotheses requires an article-level citation network specific to work produced by philosophers of science, and many covariates capturing, among other things, discipline-specific citation counts from work produced by non-philosophers. The resulting network can then be modeled using exponential random graph models (ERGMs), which are also rarely used in the bibliometric literature. The sections below clarify how we constructed this unique network dataset, and how we specified the ERGM.

First, we created a list of philosophers of science by identifying anyone who published 2 or more articles in any of the major philosophy of science journals, ${ }^{3}$ wrote a philosophy of science dissertation indexed by ProQuest Dissertations \& Theses, or was a member of the

\footnotetext{
${ }^{3}$ Philosophy of Science; British Journal for the Philosophy of Science; Studies in History and Philosophy of Science, Parts A, B, and C; Synthese; European Journal for Philosophy of Science; Journal for General Philosophy of Science; and International Studies in the Philosophy of Science. We arrived at this list by
} 
American, Canadian, or British professional associations in $2016 .{ }^{4}$ We collected the metadata for all available publications by these authors $(n=27,734)$ from the Web of Science database, ${ }^{5}$ regardless of whether the article was published in a philosophy of science journal. We refer to this collection of articles published by philosophers as Record Set 1. In addition to this data, we collected the metadata for all articles citing the articles published by philosophers of science $(n=140,918)$. We refer to this collection of citing articles as Record Set 2. We used the web of science subject classifications to group articles into 11 categories: medicine, biology, chemistry, physics, math and computer science, engineering and materials science, environment, psychology, social sciences, professional fields (e.g., law), and non-philosophy humanities. ${ }^{6}$

We used the Python package metaknowledge (McLevey and McIlroy-Young 2017) to create a citation network of the articles published by philosophers of science (24,901 nodes and 40,537 edges). Every article in this citation network was authored or co-authored by a philosopher of science. We then used metaknowledge to match each of the 24,901 articles to entries in the reference lists of the 140,918 articles in Record Set 2. From the perspective of theories of scientific trading (Yan et al. 2013; Zhu and Yan 2015), these citation counts represent disciplinary knowledge exports (e.g., work by a philosopher getting cited in medical journals).

Every time there is a match between a publication in Record Set 1 (articles by philosophers) and a citation in Record Set 2 (articles citing articles by philosophers), metaknowledge checks which of the 11 disciplinary clusters the Record Set 2 article belongs to, and then increases the citation count for the Record 1 article in the appropriate disciplinary cluster. For example, one of the papers in the philosophy Record Set 1 is Wilson and Sober's (1994) "Reintroducing group selection to the human behavioral sciences." metaknowledge then searches for the Wilson and Sober article in the reference lists of non-philosophy articles in Record Set 2. The results of this process are then used to compute 11 different citation counts for Wilson and Sober (1994); one for each of the 11 disciplinary clusters. This process is repeated for all 24,901 articles by philosophers of science.

To summarize, we constructed our final dataset as follows: (1) identify philosophers of science; (2) identify the articles these philosophers published to construct Record Set 1; (3) construct an article-level philosophy of science citation network from Record Set 1; (4) identify articles that cite the philosophy articles but are published in non-philosophy journals to construct Record Set 2; (5) use the Web of Science subject classifications to develop 11 disciplinary clusters and assign each article to one of the 11 clusters based on

Footnote 3 continued

checking journal rankings and corresponding with several highly-regarded senior philosophers of science over email.

${ }^{4}$ Unfortunately, we did not have access to the membership lists for other philosophy of science associations.

5 We had a team of 61 research assistants disambiguate authors manually. Author disambiguation is a major challenge in bibliometric research. While our approach is not perfect, current automated methods of author disambiguation are much more likely to have high rates of false positive or false negatives, depending on the approach adopted.

${ }^{6}$ The subject classifications are widely used in bibliometric research, especially when it comes to research on interdisciplinary citations and knowledge diffusion. However, they have been criticized for being somewhat arbitrary. We grouped the subject classifications into broader disciplinary clusters because (1) these more general clusters require fewer arbitrary classification decisions, and (2) having fewer categories greatly reduces the complexity of our statistical network models. 
its subject classification; (6) match the philosophy articles (Record Set 1) to the reference lists of non-philosophy articles (Record Set 2); (7) count the number of times each philosophy of science article is cited by articles from the 11 disciplinary clusters; and finally, (8) add the 11 new citation count variables as attributes of nodes in the philosophy citation network. An overview of this linked record set design is shown in Fig. 1, which maps the flow of information from Record Set 1 to Record Set 2 using our 11 aggregated subject classifications.

The left side of Fig. 1 shows the disciplinary clusters that philosophers publish in, and the right side shows the disciplinary clusters that those articles get cited in. The figure shows, as we would expect, that most citations to by philosophers come from journals in the same disciplinary field. For example, articles published in psychology journals tend to be cited by articles in other psychology journals, but they also get cited by a subset of articles published in the social sciences, medical research, and math and computer science. We have colored the citation flows for articles published in Philosophy of Science and History and Philosophy of Science journals. Roughly half of the citations to articles in these journals come from journals in different disciplines.

We developed an exponential random graph model (Lusher et al. 2012; Robins et al. 2007 ) to model the citation process within philosophy of science. This model estimates the likelihood that an article written by a philosopher of science will be cited in another article written by a philosopher of science. The linked record design outlined above enables us to use citation counts for each of the 11 disciplinary clusters as covariates in that model, which enables us to test the four hypotheses introduced at the start of the paper. We discuss the model specification below.

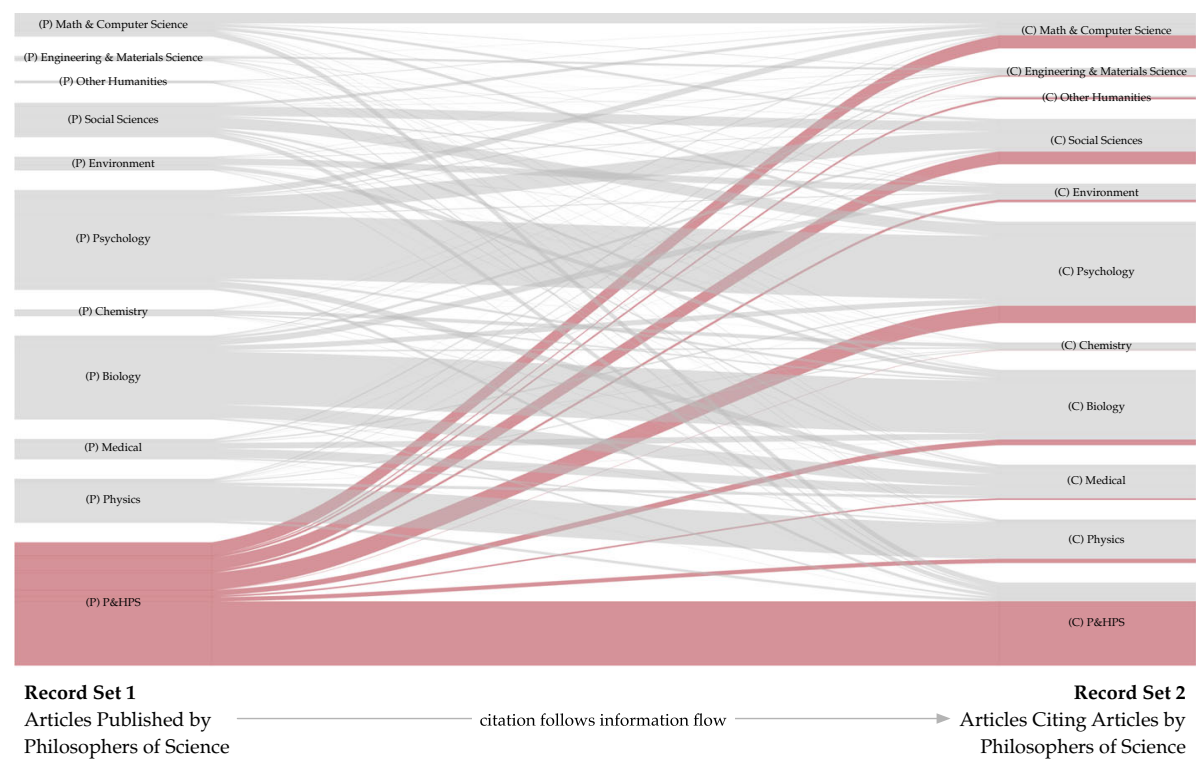

Fig. 1 Alluvial diagram of knowledge diffusion from articles published by philosophers of science to articles in 11 disciplinary clusters 


\section{Models}

ERGMs are one of the most important and widely-used classes of statistical models for networks. Broadly speaking, ERGMs have been developed with the goal of drawing inferences about the formation of network structures. One of the core theoretical ideas behind this class of models is that macro-level network structures emerge from small micro-level network patterns called "configurations" (e.g., edges, two-stars, triangles) (Lusher et al. 2012; Prell 2012; Robins 2011, 2015; Robins et al. 2007). ERGMs estimate the presence or absence of ties as a function of both lower-level endogenous network processes (e.g., transitivity, reciprocity, homophily) and exogenous factors such as attributes of nodes (Lusher et al. 2012; Prell 2012; Robins 2011, 2015; Robins et al. 2007).

For readers unfamiliar with ERGMs, it can be helpful to think of them as similar in respects to logistic regression, but with specific differences that are necessary for modeling networks statistically. ${ }^{7}$ Like a logistic regression, ERMGs estimate a binary outcome (whether or not a tie forms between two nodes) as a function of covariates. However, unlike logistic regression, which assumes independent observations, ERGMs are designed to handle the many dependencies inherent in networks by using computationally-intensive simulation techniques that are conditioned on the structure of the observed network. Unlike logistic regression, then, ERGMs can include covariates that capture endogenous network processes. For example, we can estimate the effect of transitivity, net of other covariates, on the likelihood of tie formation.

In this application, ERGMs model the likelihood of receiving a citation (i.e., a directed edge in a citation network) as a function of endogenous processes such as specialization and exogenous factors such as characteristics of the author and the journal a paper is published in. We developed two ERGMs (using the Statnet package for R, Handcock et al. 2008) to estimate the likelihood of a philosopher of science receiving a citation within the philosophy of science citation network. The first is a baseline model, and includes the endogenous covariates that we consider most relevant for capturing the dynamics of a citation network. The second is our full model, which includes a variety of exogenous variables such as the disciplinary classifications of the journals that philosophy of science articles are published in, the number of authors on a paper, the number of papers that the author or co-authors published in top-ranked philosophy of science journals, and the number of citations the article received in each of the 11 disciplinary clusters. A full list of the relevant variables is provided below.

\section{Endogenous terms in the baseline and full models}

We included endogenous terms for edges, popularity spread (geometrically weighted indegree), activity spread (outdegree), clustering (geometrically weighted edgewise shared partners), and two-paths (geometrically weighted dyadwise shared partners). The edges term estimates the probability of an edge existing between two nodes based on the number of edges that exist in the original network. It is analogous to an intercept in a regression model and is rarely interpreted. The geometrically weighted terms assume that the

\footnotetext{
7 Readers familiar with generalized linear models but unfamiliar with exponential random graph models may wish to consult the detailed comparison in Lusher et al. (2012).
} 
likelihood of additional structures existing reduces as the number increases. We describe each geometrically weighted term below.

The popularity spread term (gwidegree) allows us to control for the distribution of received citations. The term is geometrically weighted, meaning it assumes that each additional citation is less likely than the previous one. This is important because most philosophy of science articles are not highly cited. In other words, the probability of receiving 4 citations is higher than the probability of receiving 5 citations. $^{8}$ A negative effect would indicate that philosophy articles tend to receive similar levels of citations as the other philosophy articles that cite them. For example, highly cited articles are cited by other highly cited articles, and articles with few citations are cited by articles that also have few citations. Similarly, we control for the size of bibliographies using an activity spread term (gwodegree). The negative effect observed in the model indicates that articles tend to cite a similar number of papers as the articles they cite and the articles that cite them. While not substantively interesting, this term is an essential control variable.

We control for clustering in the network by using a geometrically weighted edgewise shared partner (gwesp). This term accounts for the distribution of structural triangles in the network, assuming that closing an additional triangle is less likely the more closed triangles a node is already part of. In other words, the probability of an added citation that will make an article part of a fifth triangle is less than the probability of the citation that makes it part of a fourth triangle. This term was developed by the statnet (Handcock et al. 2008) team because of the computational challenges in controlling directly for transitivity in a large network. A positive effect for gwesp suggests a higher number of closed triangles in the network, and hence greater clustering.

Finally, our last endogenous variable controls for two-paths using a geometrically weighted dyadwise shared partner (gwdsp) term. It assumes that an edge creating a twopath is less likely the more two-paths it would create. In other words, the probability that an added citation will create four two-paths is higher than the probability of creating five twopaths. A negative effect in conjunction with a positive clustering effect would indicate that two-paths tend to be closed; they appear less often than would be expected, except as part of triangles.

\section{Exogenous terms in the full model}

In addition to these endogenous terms, our full model includes exogenous variables to estimate the effects of (1) whether or not an article was published in a top-ranked journal, (2) whether or not an article was published in a journal outside of philosophy of science, (3) the number of authors on an article, (4) the total number of articles the authors previously published in the top-ranked philosophy of science journals, (5) the amount of time between publication and citation, (6) citation temporal direction (explained below), and (7) the number of citations from each of the 11 disciplinary clusters. Each of these variables is described below.

First, we have some basic controls for the types of journal in which an article is published. As Fig. 1 makes clear, philosophers of science publish in many journals that are

\footnotetext{
${ }^{8}$ Initially, this seems to contradict the well-established Matthew effect (Merton 1973)-cumulative advantage-in academic citations. However, the gwidegree term improves the goodness of fit of the model. We suspect that this is because, for most philosophy articles, the probability of being highly cited is fairly low. In other words, gwidegree helps us control for the fact the most philosophy of science articles are not highly cited. Of course, bigger fields with higher publication and citation rates may differ.
} 
outside of specialized philosophy journals. These journals all come from fields with different types of publication and citation practices. Despite these differences, we know that there are journal hierarchies in all disciplines that publish articles, and that publishing in an elite journal is different than publishing in a non-elite journal, regardless of the discipline. We control for this difference while respecting disciplinary differences in citation practices by ranking journals by their impact factors within their disciplinary contexts for each publication year. If a journal impact factor is within the top $10 \%$ in its disciplinary context in the publication year, we code it as a top-ranked journal. A positive effect for this term would indicate that an article published in a top-ranked journal is more likely to receive citations from other philosophers of science than would be expected by random chance. In addition, we added a published outside discipline term to account for the effect of publishing in journals outside of philosophy of science. A negative effect would indicate that articles published in non-philosophy of science journals are less likely to be cited by philosophers of science.

We also included two terms to control for the number of authors on a paper and the extent to which those authors published in the core philosophy of science journals leading up to the article in question. The first term is straightforward. A positive effect for number of authors would indicate that having more co-authors increases the likelihood of being cited by other philosophers of science. Philosophy of science is primarily a single-author field. Thus, having higher numbers of co-authors is an indicator of articles that are less conventional in the field. We constructed the second term-number of articles previously published in the seven core PoS journals - by searching the philosophy record set for other papers (co-)authored by the authors of any given paper. For every paper that an author previously published in one of the seven core philosophy of science journals, ${ }^{9}$ we add a score of 1. A positive effect would indicate that previous publications in core journals improve the likelihood that future publications will receive citations from other philosophers of science.

Finally, we have two terms that control for the temporal aspects of citations. First, citation temporal direction controls for the "tendency" for articles to cite articles that were published before them. Early formulations of the model suffered from unacceptable goodness of fit for mean geodesic distance because they did not have a way of handling the inability of papers to cite other papers that had not yet been written. With the rare exception of citing as-of-yet unpublished manuscripts a year or two in advance, it is impossible for authors to cite papers that don't yet exist. This problem can be ameliorated with a term that distinguishes between directional edges from later papers to earlier papers (likely) and from earlier papers to later papers (highly unlikely). No suitable constraint or term was found in any of the ERGM packages available as of this paper's publication date; as such, we used the ergm.userterms package (Hunter et al. 2013) to construct our own term that controls for the tendency of papers to cite older papers. A positive effect indicates that papers are more likely to cite papers older than themselves. This term proved essential to improving the goodness of fit to the point where the observed network and the simulated networks were in near-perfect agreement.

The second temporal term, absolute age difference, captures the amount of time between the publication of an article and its citations. A positive effect would indicate that

\footnotetext{
9 The top-ranked journal variable indicates whether or not the article was published in a journal whose impact factor is within the top $10 \%$ within its disciplinary context. The seven core philosophy of science journals are not identified based on their impact factors. Instead, they are the journals that philosophers of science have indicated are the major specialized journals within their field.
} 
papers are more likely to receive citations from papers that are published shortly thereafter, and less likely to receive citations from papers published far in the future.

The disciplinary terms in the model enable us to assess the relationships between citations from philosophers of science and citations from each of the 11 disciplinary clusters. A positive effect for any of these 11 variables would indicate that, net of other factors, citations from non-philosophers of science increase the likelihood of also receiving citations from other philosophers of science, and would therefore support the cumulative advantage hypothesis. A negative effect would suggest that receiving citations from outside philosophy of science reduces the likelihood of being cited by other philosophers, and would therefore support the citation penalty hypothesis. Minimal or negligible effects would support the indifference hypothesis, as increased citations from outside philosophy of science neither increase or decrease the likelihood of being cited by philosophers. Support for the field-specific hypothesis would show up as a mix of positive, negative, or no effect across disciplinary clusters.

\section{Results}

Our baseline model includes the endogenous properties we considered most important to a disciplinary citation network, including the number of citations in the network (edges), the distributions of giving and receiving citations (popularity spread and activity spread), and the propensity for clustering and two-paths. ${ }^{10}$ The results of the baseline model 1 are presented in Table 1.

Our baseline model does a reasonably good job of capturing the dynamics of citations in philosophy of science despite including only endogenous covariates. All were statistically significant. The popularity spread term's negative coefficient suggests that articles tend to have a similar number of citations as the articles they cite. The strongly positive clustering term indicates a high number of transitive triads, and the much smaller negative two-path term suggests a weak but significant tendency away from unclosed triads. Together, these two effects suggest that philosophy papers often cite papers in a relevant area of discussion, and they tend not to cite papers that do not cite other relevant papers. In other words, philosophers tend to cite papers within the pool of papers relevant to their topic; our baseline model is primarily capturing specialization within philosophy of science. ${ }^{11}$ This is exactly what a good baseline model of a citation network should capture.

We created goodness of fit diagnostic plots - shown in Fig. 2-for the baseline model. The basic idea in assessing goodness of fit in ERGMs is whether or not simulations conditional on the observed network captures structural features (e.g., minimum geodesic distance) that were not actually modeled (Hunter et al. 2008; Koskinen and Snidjers

\footnotetext{
${ }^{10}$ By including these terms, we ensure that significant two-path and clustering effects are separate from the functional dependencies that arise from edges, popularity spread, and activity spread.

11 Of course, we do not mean to suggest that this is the only reason for citation clustering. There are complex reasons for why authors cite some authors and not others. However, when looking at patterns within and across intellectual fields, decisions about which author or paper to cite are most likely to come into play within some smaller subset of authors and articles, not the entire field. For example, if I am networks researcher making a decision about who to cite for an overview of statistical approaches to network analysis, I am more likely to cite someone who is actually doing work on statistical models for networks than someone working in another area of network science. Within the subset of authors working on statistical network models, I may make citation decisions in ways that favour people I know, or who have cited me, etc. Given the type of models and analysis in this paper, we think it is reasonable to assume that the citation clustering we see if primarily driven by specialization, even if individual citation decisions between one author or another are shaped by other considerations.
} 
Table 1 ERGM results

Likelihood of PoS citation

(1)

(2)

\begin{tabular}{|c|c|c|}
\hline Edges & $7.177 * * *$ & $-17.535^{* * *}$ \\
\hline Popularity spread & $-3.406^{* * * *}$ & $-2.507 * * *$ \\
\hline Activity spread & $-2.522 * * *$ & $-1.658 * * *$ \\
\hline Clustering $(\alpha=.7)$ & $3.983 * * *$ & $5.236^{* * *}$ \\
\hline Two-paths $(\alpha=.7)$ & $-0.169 * * *$ & $-0.141 * * *$ \\
\hline Top-ranked journal & & 0.072 \\
\hline Journal outside PoS & & $-0.187 * * *$ \\
\hline Number of authors & & 0.001 \\
\hline No. of articles previously published in the seven core PoS journals & & $0.016 * * *$ \\
\hline Citation temporal direction & & $10.946 * * *$ \\
\hline Absolute age difference & & $-0.065^{* * *}$ \\
\hline \multicolumn{3}{|l|}{ Citations from disciplinary clusters: } \\
\hline Biology & & 0.0002 \\
\hline Chemistry & & $-0.005^{* * *}$ \\
\hline Engineering and materials science & & 0.001 \\
\hline Environment & & $0.003 * * *$ \\
\hline Humanities & & $0.054 * * *$ \\
\hline Math and computer science & & $0.007 * * *$ \\
\hline Medical science & & $0.002 * * *$ \\
\hline Physics & & $-0.001 * * *$ \\
\hline Professional fields & & -0.001 \\
\hline Psychology & & $-0.001 * * *$ \\
\hline Social science & & 0.0003 \\
\hline AIC & $842,182.700$ & $743,550.900$ \\
\hline $\mathrm{BIC}$ & $842,273.900$ & $743,952.300$ \\
\hline
\end{tabular}

$* p<.05 ; * * p<.01 ; * * * p<.001$

2013). ${ }^{12}$ The plots show how well the observed network is described by purely endogenous effects. The simulated networks were suitably well matched to the observed distributions across indegree, outdegree, edgewise shared partners. The model slightly underestimated indegree and outdegree distributions for values of 1 and 2, and slightly overestimated for values from 4 to 9 . This suggests that our model had some issue capturing the behavior of these distributions at those values. This might indicate that an unmeasured variable has a particularly strong effect on papers with indegrees and outdegrees at these values, or that there may be a shift in behavior at these values that the geometrically-weighted terms cannot perfectly capture. The model overestimated the proportion of edges that were not part of a transitive triad and slightly underestimated the proportions in 1 or 2 transitive triads. All other values and terms were within the estimated margins of error for the

12 For readers unfamiliar with ERGMs, in the two goodness of fit plots in this paper, goodness of fit should considered good if the thick black lines (observed statistics) are aligned with the simulations (the boxplots). 


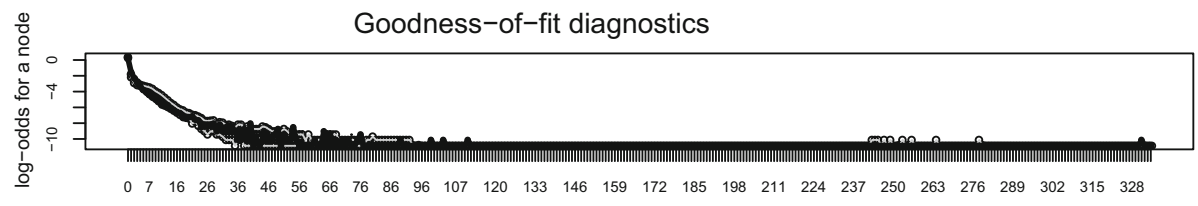

in degree
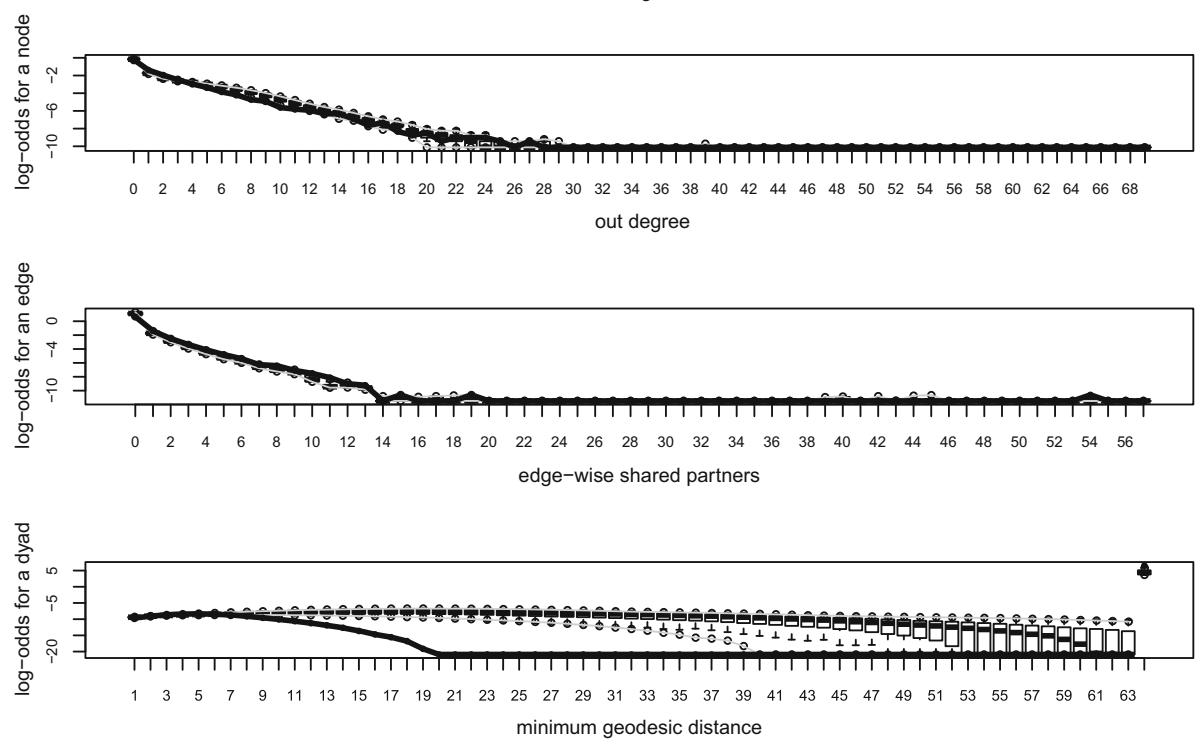

Fig. 2 Goodness-of-fit diagnostic plots for the base model with endogenous terms only. The thick black lines represent observed network statistics. Boxplots represent distributions from the simulated networks

observed network. As previously noted, the goodness of fit is not acceptable for minimum geodesic distance. We resolved this issue by including the citation temporal direction term in the full model.

Our full model kept the terms used in the baseline model and included new exogenous terms controlling for temporal direction of citations, difference in ages between citing and cited papers, whether or not an article was published in a top-ranked philosophy of science journal, whether or not an article was published in a journal outside of philosophy of science, the number of authors, total number of articles the authors previously published in the seven core philosophy of science journals. We also included exogenous terms for citation counts in each of the 11 disciplinary cultures. The results are reported in Table 1.

All of our endogenous effects remained statistically significant. Their estimated coefficients remained similar, with the exception of the clustering term, which became slightly stronger. This means that, after controlling for exogenous effects, we see an even stronger tendency towards closing transitive citation triads. Again, this almost certainly reflects specialization in the philosophy of science citation network. The exogenous control variables show that being an older paper (i.e., having longer to accumulate citations), being published in a top philosophy of science journal, ${ }^{13}$ having a history of publishing other

13 The positive effect for this term may be due to several non-exclusive mechanisms. Papers in major journals may be more visible, thus receiving greater attention. Authors may choose to cite papers from major journals on the premise that they provide stronger evidence than papers from less prestigious journals. The 
papers in core philosophy of science journals, and having co-authors all have positive effects on receiving a citation. The negative coefficient for the absolute difference in age between citing and cited papers indicates that papers tend to cite papers that are similar in age. In other words, authors tend to cite newer work more frequently than a large body of classics.

Publishing in a non-philosophy of science journal has a negative and significant effect on receiving citations from philosophers of science. One simple reason for this effect could be that papers in non-philosophy of science journals are less visible to philosophers of science or are less relevant to disciplinary discussions, and thus are cited less. Other possibilities include that traditional philosophers of science view publishing in science journals as not maintaining a critical distance from the scientific communities or not meeting high philosophical standards. Of course, our model cannot differentiate between these mechanisms nor determine if all are at work.

The terms for citations in each of the 11 disciplinary clusters enable us to evaluate our hypotheses about the relationship between philosophy of science and the sciences. We found positive effects for receiving citations from environment, humanities, math and computer science, and medical papers. Conversely, we found negative effects for receiving citations from chemistry, physics, and psychology papers. None of the other terms indicated significant effects. While the observed mix of positive and negative effects across the disciplines could be interpreted as support for the field-specific hypothesis, most effects are extremely small. Depending on the case, we would be looking at hundreds of citations from scientists costing a single citation from a philosopher. Presumably few philosophers would trade 100 citations from non-philosophers for a single citation from another philosopher. Therefore, we consider this to be better evidence for the indifference hypothesis. Receiving citations from any of these 11 disciplinary clusters does not result in any meaningful citation boosts or penalties.

We compared the goodness of fit for our full model, shown in Fig. 3, against the goodness of fit for the baseline model, shown in Fig. 2. The model's estimation of the original network's distribution has minor improvements for in-degree, out-degree, and edge-wise shared partners, and major improvements for minimum geodesic distance. Both the base and full models are able to accurately reproduce global features of the observed network, but the full model is a considerably better fit.

\section{Discussion and conclusion}

As stated at the beginning of this paper, we consider highly general statements about the siloed or webbed nature of disciplinary communication and boundaries to be counterproductive. Instead, the goal of this work is to contribute to a line of research that focuses on the nature of specific disciplinary boundaries, and on the relationship between disciplinary boundaries and citation dynamics. To that end, our goal in this paper was to develop statistical network models to test hypotheses about how publishing in and being cited by articles published in science journals impact philosophers' positions within their disciplinary citation network.

We found that philosophers of science publish regularly outside of their discipline, and that much of this philosophical work gets cited by articles in science journals. However,

Footnote 13 continued

major journals may get the highest quality submissions from the field, allowing them to pick the best, which are then subsequently recognized by other philosophers in the form of citations. 

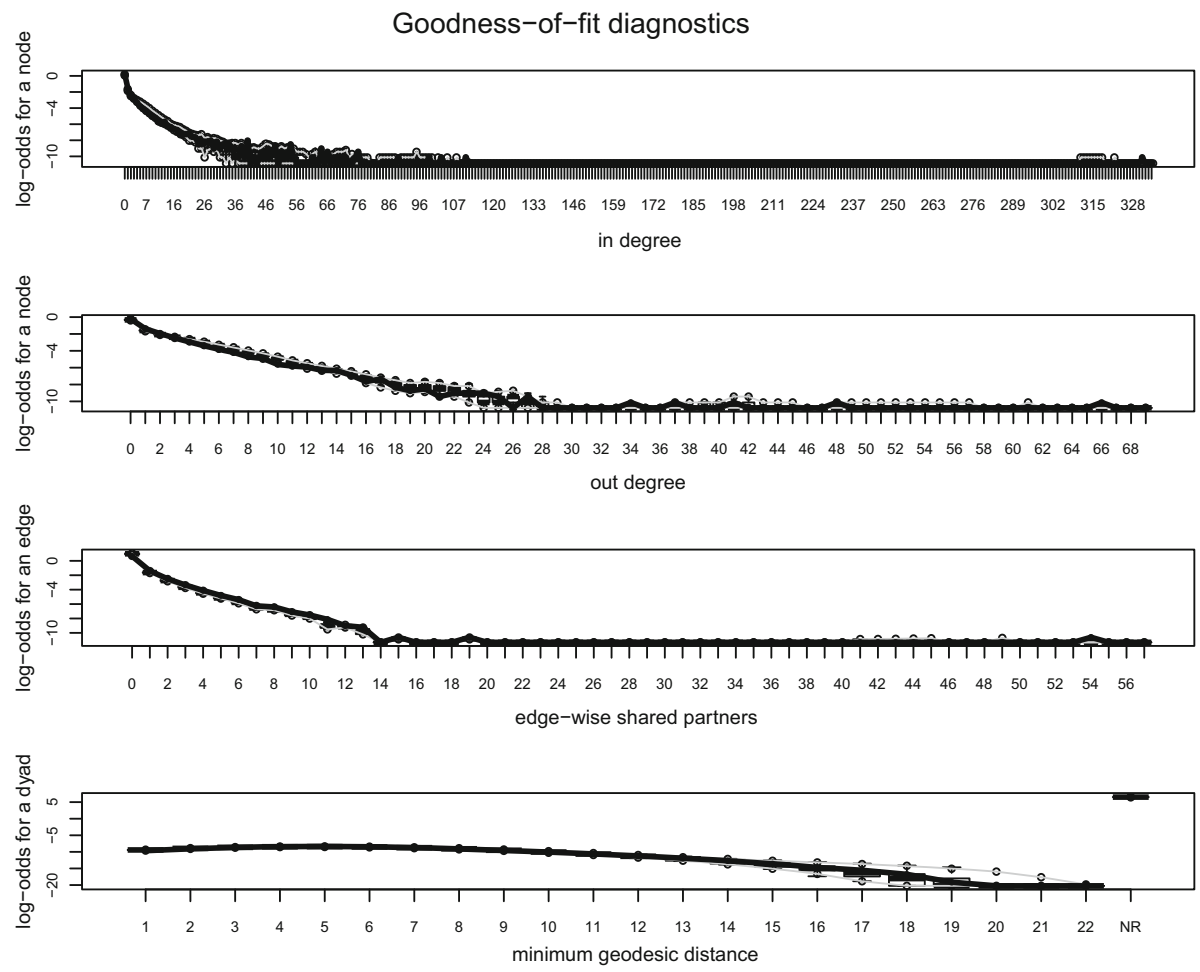

Fig. 3 Goodness-of-fit diagnostic plots for the full model with endogenous and exogenous terms. The thick black lines represent observed network statistics. Boxplots represent distributions from the simulated networks

these interdisciplinary exchanges do not result in philosophers gaining or losing influence within their disciplinary context. These findings are broadly supportive of the "indifference hypothesis:" net of other factors, philosophers' successes outside of their own disciplinary context are largely irrelevant to their standing within philosophy of science.

Somewhat paradoxically, these findings can be interpreted as broadly consistent with both general perspectives on knowledge diffusion and disciplinary boundaries introduced at this start of this article. As we have shown, an enormous amount of the work done by philosophers of science is published in journals from other disciplines, and this work is cited outside of philosophy of science. Even more telling, roughly half of the citations to articles published in philosophy of science and history and philosophy of science journals come from articles published outside the discipline (see the bottom left side of Fig. 1). This seems to us to be perfectly consistent with other bibliometric challenges to the "disciplines are silos" argument.

Despite this seemingly clear evidence against the siloed disciplines argument, philosophers of science simultaneously display insularity. Collectively, they appear indifferent to philosophical work that appears in journals outside of their own field, and philosophers of science who are cited by non-philosophers do not see a corresponding increase in their citations from philosophers. It seems that, collectively, philosophers of science are largely unaware of and indifferent towards the publication of and citation of philosophical work in non-philosophy journals. This evidence of insularity is aligned with 
the general criticism of disciplines as inward-looking silos. In this sense, it makes sense to think of philosophy of science and the sciences as separate worlds, and philosophers often export their work to the non-philosophy worlds, but what happens in the non-philosophy worlds doesn't have an impact on what happens in philosophy of science.

This is yet another reason why we think that empirical work on the boundaries between and intellectual reputations within specific disciplines is more useful than high-level arguments about the state of knowledge diffusion and communication across all disciplinary boundaries. The simultaneous evidence of interdisciplinarity and insularity in philosophy of science is, in our view, consistent with the argument from scientific trading theory that some disciplines are more self-contained than others while still being embedded in a massive intellectual network of linked disciplines (Boyack et al. 2005; Börner et al. 2003; Börner et al. 2012; Chen and Hicks 2004; Leydesdorff and Rafols 2009; Moody and Light 2006; Skupin et al. 2013). If philosophers of science were genuinely at odds with the sciences they study, we would see evidence of a citation penalty for philosophical work that is published in, or receives a lot of attention from, scientists. If, on the other hand, they perceive only weak and relatively unimportant disciplinary boundaries between their work and that of the sciences, then we would expect to see evidence of a citation boost. However, our results suggest that philosophy of science is a field in which philosophers are neither rewarded nor penalized for engaging with and being recognized by other fields. It is simultaneously open (interdisciplinary without penalty) and closed (interdisciplinary without reward).

Of course, this simultaneous interdisciplinarity and insularity-philosophers neither gain nor lose when their work is exported to the sciences they study-does not mean that there are no costs for those who want to do work that is engaged with scientists. Doing scientifically engaged work is easier for philosophers who work in departments that explicitly value interdisciplinary scholarship. Our work is consistent with the idea that philosophers who do not work in such departments and who do want to do scientifically engaged work must have dual philosophical and scientific careers, which is certainly a high price to pay. However, this aspect of the problem is institutional and cannot be adequately addressed with models of citation dynamics. Instead, we address it in separate papers that analyze data from surveys and semi-structured interviews with philosophers of science.

\section{References}

Bettencourt, L., Kaiser, D., Kaur, J., Castillo-Chavez, C., \& Wojick, D. (2008). Population modeling of the emergence and development of scientific fields. Scientometrics, 75(3), 495-518.

Börner, K., Chen, C., \& Boyack, K. (2003). Visualizing knowledge domains. Annual Review of Information Science and Technology, 37(1), 179-255.

Börner, K., Klavans, R., Patek, M., Zoss, A., Biberstine, J., Light, R., et al. (2012). Design and update of a classification system: The UCSD map of science. PLoS ONE, 7(7), e39464.

Boyack, K., Klavans, R., \& Börner, K. (2005). Mapping the backbone of science. Scientometrics, 64(3), $351-374$.

Campbell, D. (1969). Ethnocentrism of disciplines and the fish-scale model of omniscience. Interdisciplinary relationships in the social sciences, 328, 348.

Chen, S., Arsenault, C., Gingras, Y., \& Larivière, V. (2014). Exploring the interdisciplinary evolution of a discipline: The case of biochemistry and molecular biology. Scientometrics, 102(2), 1307-1323.

Chen, S., Arsenault, C., \& Larivière, V. (2015). Are top-cited papers more interdisciplinary? Journal of Informetrics, 9(4), 1034-1046.

Chen, C., \& Hicks, D. (2004). Tracing knowledge diffusion. Scientometrics, 59(2), 199-211. 
Coccia, M., \& Bozeman, B. (2016). Allometric models to measure and analyze the evolution of international research collaboration. Scientometrics, 108(3), 1065-1084.

Coccia, M., \& Wang, L. (2016). Evolution and convergence of the patterns of international scientific collaboration. Proceedings of the National Academy of Sciences, 113(8), 2057-2061.

Collins, R. (2009). The sociology of philosophies. Cambridge: Harvard University Press.

Collins, H. (2011). Language and practice. Social Studies of Science, 41(2), 271-300.

Collins, H., Evans, R., \& Gorman, M. (2010). Trading zones and interactional expertise. In M. Gorman (Ed.), Trading zones and interactional expertise (pp. 7-25). Cambridge: MIT Press.

Crane, D. (1972). Invisible colleges: Diffusion of knowledge in scientific communities. Chicago: University of Chicago Press.

Cronin, B., \& Meho, L. I. (2008). The shifting balance of intellectual trade in information studies. Journal of the American Society for Information Science and Technology, 59(4), 551-564.

Cronin, B., \& Pearson, S. (1990). The export of ideas from information science. Journal of information science, 16(6), 381-391.

Cronin, B., \& Sugimoto, C. (2014). Beyond bibliometrics: Harnessing multidimensional indicators of scholarly impact. Cambridge: MIT Press.

Fanelli, D., \& Glänzel, W. (2013). Bibliometric evidence for a hierarchy of the sciences. PLoS ONE, 8(6), e66938.

Fehr, C., \& Plaisance, K. S. (2010). Socially relevant philosophy of science: An introduction. Synthese, 177(3), 301-316.

Frodeman, R. (Ed.). (2010). The Oxford handbook of interdisciplinarity. Oxford: Oxford University Press.

Galison, P. (1997). Image and logic: A material culture of microphysics. Chicago: University of Chicago Press.

Gao, X., \& Guan, J. (2011). Network model of knowledge diffusion. Scientometrics, 90(3), 749-762.

Gorman, M. (2002). Levels of expertise and trading zones: A framework for multidisciplinary collaboration. Social Studies of Science, 32(5-6), 933-938.

Gorman, M. (2010). Trading zones and interactional expertise: Creating new kinds of collaboration. Cambridge: MIT Press.

Gross, N. (2002). Becoming a pragmatist philosopher: Status, self-concept, and intellectual choice. American Sociological Review, 67(1), 52-76.

Gross, N. (2009). Richard Rorty: The making of an American philosopher. Chicago: University of Chicago Press.

Handcock, M., Hunter, D., Butts, C., Goodreau, S., \& Morris, M. (2008). Statnet: Software tools for the representation, visualization, analysis and simulation of network data. Journal of Statistical Software, 24(1), 1548 .

Herrera, M., Roberts, D. C., \& Gulbahce, Natali. (2010). Mapping the evolution of scientific fields. PLoS ONE, 5(5), e10355.

Hunter, D., Goodreau, S., \& Handcock, M. (2008). Goodness of fit of social network models. Journal of the American Statistical Association, 103(481), 248-258.

Hunter, D., Goodreau, S., \& Handcock, M. (2013). Ergm. Userterms: A template package for extending statnet. Journal of Statistical Software, 52(2), i02.

Ilhan, A. (2013). The growth of the design disciplines in the united states 1984-2010. Ph.D. thesis. Washington State University.

Jacobs, J. (2014). In defence of disciplines: Interdisciplinarity and specialization in the research university. Chicago: Chicago University Press.

Jacobs, J., \& Frickel, S. (2009). Interdisciplinarity: A critical assessment. Annual Review of Sociology, 35, 43-65.

Jiang, S., Gao, Q., Chen, H., \& Roco, M. (2015). The roles of sharing, transfer, and public funding in nanotechnology knowledge-diffusion networks. Journal of the Association for Information Science and Technology, 66(5), 1017-1029.

Klein, J. T. (1990). Interdisciplinarity: History, theory, and practice. Detroit: Wayne State University Press.

Koskinen, J., \& Snidjers, T. (2013). Simulation, estimation, and goodness of fit. eds. Dean Lusher, Koskinen Johan, and Garry Robins.: 141-66.

Kreuzman, H. (2001). A co-citation analysis of representative authors in philosophy: Examining the relationship between epistemologists and philosophers of science. Scientometrics, 50(3), 525-539.

Lamont, M. (2009). How professors think: Inside the curious world of academic judgment. Cambridge: Harvard University Press.

Larivière, V., \& Gingras, Y. (2014). Measuring interdisciplinarity. In B. Cronin \& C. Sugimoto (Eds.), Beyond bibliometrics: Harnessing multidimensional indicators of scholarly impact (pp. 187-200). Cambridge: MIT. 
Larivière, V., Sugimoto, C. R., \& Cronin, B. (2012). A bibliometric chronicling of library and information science's first hundred years. Journal of the Association for Information Science and Technology, 63(5), 997-1016.

Leydesdorff, L., \& Rafols, I. (2009). A global map of science based on the Isi subject categories. Journal of the American Society for Information Science and Technology, 60(2), 348-362.

Light, R., \& Adams, J. (2016). Knowledge in motion: The evolution of HIV/Aids research. Scientometrics, 107(3), 1227-1248.

Liu, X., Jiang, S., Chen, H., Larson, C. A., \& Roco, M. C. (2015). Modeling knowledge diffusion in scientific innovation networks: An institutional comparison between China and US with illustration for nanotechnology. Scientometrics, 105(3), 1953-1984.

Lockett, A., \& McWilliams, A. (2005). The balance of trade between disciplines: Do we effectively manage knowledge? Journal of Management Inquiry, 14(2), 139-150.

Lungeanu, A., Huang, Y., \& Contractor, N. (2014). Understanding the assembly of interdisciplinary teams and its impact on performance. Journal of Informetrics, 8(1), 59-70.

Lusher, D., Koskinen, J., \& Robins, G. (2012). Exponential random graph models for social networks: Theory, methods, and applications. Cambridge: Cambridge University Press.

McLevey, J., \& McIlroy-Young, R. (2017). Introducing Metaknowledge: Software for computational research in information science, network analysis, and science of science. Journal of Informetrics, 11(1), 176-197.

Merton, R. (1973). The sociology of science: Theoretical and empirical investigations. Chicago: University of Chicago Press.

Moody, J., \& Light, R. (2006). A view from above: The evolving sociological landscape. The American Sociologist, 37(2), 67-86.

Panofsky, A. (2014). Misbehaving science: Controversy and the development of behavior genetics. Chicago: University of Chicago Press.

Prell, C. (2012). Social network analysis: History, theory, \& methodology. Thousand Oaks: Sage.

Price de Solla, D. J. (1963). Little science, big science. New York: Columbia University Press.

Robins, G. (2011). Exponential random graph models for social networks. In eds. John Scott and Peter Carrington.: 484-500.

Robins, G. (2015). Doing social network research: Network-based research design for social scientists. Thousand Oaks: SAGE.

Robins, G., Pattison, P., Kalish, Y., \& Lusher, D. (2007). An introduction to exponential random graph (P*) models for social networks. Social Networks, 29(2), 173-191.

Rojas, F. (2007). From black power to black studies: How a radical social movement became an academic discipline. Baltimore: JHU Press.

Škerlavaj, M., Dimovski, V., \& Desouza, K. C. (2010). Patterns and structures of intra-organizational learning networks within a knowledge-intensive organization. Journal of Information Technology, 25(2), 189-204.

Skupin, A., Biberstine, J., \& Börner, K. (2013). Visualizing the topical structure of the medical sciences: A self-organizing map approach. PLoS ONE, 8(3), e58779.

Small, M. L. (1999). Department conditions and the emergence of new disciplines: Two cases in the legitimation of African-American studies. Theory and Society, 28(5), 659-707.

Snow, C. P. (1959). The two cultures. Cambridge: Cambridge University Press.

Stigler, S. M. (1994). Citation patterns in the journals of statistics and probability. Statistical Science, 9 , 94-108.

Su, C., \& Contractor, N. (2011). A multidimensional network approach to studying team members' information seeking from human and digital knowledge sources in consulting firms. Journal of the American Society for Information Science and Technology, 62(7), 1257-1275.

Turk-Bicakci, L. (2007). The development of social movement programs and departments in higher education: Women's and ethnic studies from 1975 to 2000. Ph.D. thesis. University of California Riverside.

Van Leeuwen, T., \& Robert, T. (2000). Interdisciplinary dynamics of modern science: Analysis of crossdisciplinary citation flows. Research Evaluation, 9(3), 183-187.

Vitanov, N. K, \& Ausloos, M. R. (2012). Knowledge epidemics and population dynamics models for describing idea diffusion. In: A. Scharnhorst, K. Börner \& P. van den Besselaar (Eds.), Models of science dynamics: Encounters between complexity theory and information sciences (pp. 69-125). Berlin: Springer.

Wilson, D. S., \& Sober, E. (1994). Reintroducing group selection to the human behavioral sciences. Behavioral and Brain Sciences, 17(04), 585-608. 
Wray, B., \& Bornmann, L. (2015). Philosophy of science viewed through the lense of 'referenced publication years spectroscopy' (RPYS). Scientometrics, 102(3), 1987-1996.

Yan, E. (2014). Finding knowledge paths among scientific disciplines. Journal of the Association for Information Science and Technology, 65(11), 2331-2347.

Yan, E., Ding, Y., Cronin, B., \& Leydesdorff, L. (2013). A bird's-eye view of scientific trading: Dependency relations among fields of science. Journal of Informetrics, 7(2), 249-264.

Yu, G., Wang, M.-Y., \& Da-Ren, Y. (2010). Characterizing knowledge diffusion of nanoscience \& nanotechnology by citation analysis. Scientometrics, 84(1), 81-97.

Zhu, Y., \& Yan, E. (2015). Dynamic subfield analysis of disciplines: An examination of the trading impact and knowledge diffusion patterns of computer science. Scientometrics, 104(1), 335-359.

\title{
Affiliations
}

\section{John McLevey ${ }^{1}$ (D) Alexander V. Graham ${ }^{2} \cdot$ Reid Mcllroy-Young ${ }^{3}$ • Pierson Browne ${ }^{2} \cdot$ Kathryn S. Plaisance ${ }^{4}$}

\author{
Alexander V. Graham \\ a24graha@uwaterloo.ca \\ Reid McIlroy-Young \\ reidmcy@uchicago.edu \\ Pierson Browne \\ pbrowne@uwaterloo.ca \\ Kathryn S. Plaisance \\ kplaisan@uwaterloo.ca
}

1 Department of Knowledge Integration, Department of Sociology and Legal Studies, University of Waterloo, Waterloo, ON, Canada

2 Department of Sociology and Legal Studies, University of Waterloo, Waterloo, ON, Canada

3 Computational Social Science Program, Division of the Social Sciences, University of Chicago, Chicago, IL, USA

4 Department of Knowledge Integration, Department of Philosophy, University of Waterloo, Waterloo, ON, Canada 